\title{
Aortic Valve Leaflet Disruption: A Severe Complication of Impella 5.5
}

\author{
Alexander Ghannam ${ }^{1}$, Manabu Takebe ${ }^{2}$, Scott Tatum ${ }^{2}$, and John Pirris ${ }^{2}$ \\ ${ }^{1}$ University of Florida Health Science Center Jacksonville \\ ${ }^{2}$ University of Florida College of Medicine - Jacksonville
}

September 11, 2020

\begin{abstract}
A 73-year-old male with a history of severe coronary artery disease and prior CABG presented with chest pain and elevated troponins. His workup revealed an ejection fraction of $10-15 \%$ and severe native coronary disease as well as stenosis of bypass grafts. He underwent high-risk redo CABG with Impella 5.5 (Abiomed, Danvers, MA) placement. The Impella was removed on postoperative day eight at which time he went into cardiogenic shock from aortic valve leaflet disruption and severe aortic insufficiency. Given that this patient had severe aortic insufficiency and no calcium deposits around the aortic valve annulus a multidisciplinary heart team decided he would be best served by a surgical aortic valve replacement. He was taken back to the operating room for a surgical aortic valve and intra-aortic balloon pump. His postoperative course was complicated by pneumonia, sepsis, and renal failure requiring continuous renal replacement therapy. He was discharged to rehab after 42 days.
\end{abstract}

\section{Aortic Valve Leaflet Disruption: A Severe Complication of Impella 5.5}

Requiring Surgical Aortic Valve

Alexander D. Ghannam ${ }^{1}$, Manabu Takebe ${ }^{2}$, Scott Tatum ${ }^{2}$, John Pirris ${ }^{2}{ }^{1}$ Surgery, University of Florida, Jacksonville, FL. ${ }^{2}$ Cardiothoracic Surgery, University of Florida, Jacksonville, FL

\section{Addresses for Correspondence}

Alexander Ghannam MD

University of Florida College of Medicine - Jacksonville

Department of Surgery 655 West 8th Street 8th Floor, Clinical Center Jacksonville, FL 32209 Fax: (904)

244-4867 Mobile: (313) 671-1666 Email: alex@ghannam.us

Manabu Takebe MD

University of Florida College of Medicine - Jacksonville

Division of Cardiothoracic Surgery

655 West 8th Street 5th Floor, Ambulatory Care Center Jacksonville, FL 32209

Phone: (904) 244-3418

Fax: (904) 244-4867

Email: Manabu.Takebe@jax.ufl.edu

Scott Tatum PA-C 
University of Florida College of Medicine - Jacksonville

Division of Cardiothoracic Surgery

655 West 8th Street 5th Floor, Ambulatory Care Center Jacksonville, FL 32209

Phone: (904) 244-3418

Fax: (904) 244-4867

Email: Scott.Tatum@jax.ufl.edu

John Pirris MD

University of Florida College of Medicine - Jacksonville

Division of Cardiothoracic Surgery

655 West 8th Street 5th Floor, Ambulatory Care Center Jacksonville, FL 32209

Phone: (904) 244-3418

Fax: (904) 244-4867

Email: John.Pirris@jax.ufl.edu

Funding: None

Key Words: Impella 5.5, Heart Failure, High Risk CABG, Aortic Valve Leaflet Injury, Aortic Insufficiency

Abstract: A 73-year-old male with a history of severe coronary artery disease and prior CABG presented with chest pain and elevated troponins. His workup revealed an ejection fraction of 10-15\% and severe native coronary disease as well as stenosis of bypass grafts. He underwent high-risk redo CABG with Impella 5.5 (Abiomed, Danvers, MA) placement. The Impella was removed on postoperative day eight at which time he went into cardiogenic shock from aortic valve leaflet disruption and severe aortic insufficiency. Given that this patient had severe aortic insufficiency and no calcium deposits around the aortic valve annulus a multidisciplinary heart team decided he would be best served by a surgical aortic valve replacement. He was taken back to the operating room for a surgical aortic valve and intra-aortic balloon pump. His postoperative course was complicated by pneumonia, sepsis, and renal failure requiring continuous renal replacement therapy. He was discharged to rehab after 42 days.Introduction: Impella (Abiomed, Danvers, MA) is a mechanical support device designed for short term ( $<14$ days) unloading the left ventricle in patients with cardiogenic shock. ${ }^{1,2}$ Based on the size chosen $(2.5,5.0$, or 5.5$)$, the device is placed either percutaneously or via arteriotomy and ultimately seated across the aortic valve. Impella has established itself as an essential tool for multidisciplinary heart teams to treat patients with acute myocardial infarction, high risk coronary artery bypass grafting (CABG) or percutaneous coronary intervention (PCI), left ventricular dysfunction, and ultimately cardiogenic shock. ${ }^{1}$ Risks associated with Impella are mostly related to vascular access, however other significant complications include stroke, myocardial infarction, acute renal dysfunction/failure, hemolysis, bleeding, and aortic valve injury. ${ }^{2}$ There are reports of aortic valve injury resulting in aortic regurgitation after Impella 2.5 and 5.0 use, however it is unclear what the true incidence of this complication is. ${ }^{3-5}$ Materials and Methods: High-risk redo CABG with Impella 5.5 placement via direct aortic approach. Redo sternotomy, aortic valve replacement, and intra-aortic balloon pump (IABP) placement for perforated left coronary cusp on postoperative day eight. For the purposes of drafting this manuscript, all activities were performed in accordance with the ethical standards of the institutional and/or national research committee.

Results: A 73 year-old-male with a history of hypertension, hyperlipidemia, diabetes, chronic kidney disease, tobacco abuse, and coronary artery disease with previous CABG in 1995 presented with chest pain, shortness of breath, and cough for three days. Initial evaluation revealed elevated troponins and he was taken urgently for a left heart catheterization which revealed diffuse native coronary disease, patent left internal mammary 
to a diagonal, and two saphenous vein grafts with minimal blood flow. Initial transthoracic echocardiography (TTE) demonstrated ejection fraction (EF) of 10-15\% and no structural heart disease. After an extensive workup revealed significant hibernating myocardium, a multidisciplinary heart team determined that he would be best served by high-risk redo CABG with a plan for placement of Impella 5.5 via a direct aortic approach for support postoperatively.

The CABG was carried out in standard fashion and the Impella was placed through a graft into the ascending aorta without the use of a wire while on full cardiopulmonary bypass after the cross clamp had been removed. Once the device appeared to be in an appropriate position based on TTE guidance the graft was tunneled out superior to the right clavicle. His initial course was unremarkable and he was extubated on postoperative day one. TTE on postoperative day three demonstrated EF of $40 \%$ and appropriate positioning of the device. On postoperative day eight he underwent Impella removal at bedside at which time the device was turned down to P0 and all other safety standards were followed according to the clinical manual. ${ }^{2}$ A few hours after removal, he experienced a drop in his cardiac index from 3.1 to $1.9 \mathrm{~L} / \mathrm{min} / \mathrm{m}^{2}$. Repeat TTE demonstrated severe aortic regurgitation with a laceration of the left coronary cusp. A multidisciplinary heart team was again employed to discuss aortic valve replacement from surgical or transcatheter (TAVR) approach. Due to a lack of calcium around the aortic valve annulus and concern for possible valve migration, ${ }^{6}$ the heart team and the patient's family decided to pursue surgical aortic valve.

He was taken back to the OR on postoperative day eight for a $21 \mathrm{~mm}$ mechanical valve and an IABP placement. The IABP was removed following day, however he developed purulent respiratory secretions and had trouble being weaned from the ventilator. Bronchoalveolar lavage revealed pseudomonas pneumonia. On postoperative day 18 he developed worsening renal function eventually requiring continuous renal replacement on day 21. He underwent tracheostomy and percutaneous gastrostomy tube placement on day 25 and was able to be weaned from the ventilator and transitioned from continuous renal replacement to hemodialysis. He was discharged to a rehab facility on post-operative day 42.

Conclusion: While Impella 5.5 is an integral tool in the armamentarium of heart teams caring for patients in cardiogenic shock, it is not without its pitfalls. It is unclear whether the valve was injured on insertion or removal because device causes significant ultrasound artifact on echocardiography. ${ }^{3}$ Although it is not required in the manual, we believe device manipulation should be conducted with a guidewire under echocardiographic and/or fluoroscopic guidance regardless of approach. Our team ultimately chose to use a surgical aortic valve to repair this lesion because of the paucity of data regarding use of TAVR for aortic insufficiency. Many team members also expressed concern about the risk of valve migration with minimal calcification around the aortic valve.

Key Clinical Message: While Impella is an excellent device for managing patients in cardiogenic shock, extreme care must be taken when manipulating the device to prevent complications.

Conflict of Interest: The authors have no conflicts of interest to disclose.

\section{Author Contributions:}

Alexander D. Ghannam drafted the article. Scott Tatum and Manabu Takebe edited and approved the article. John Pirris provided concept and design as well as critical revision.

\section{Resources:}

1. Glazier, J. J., \& Kaki, A. (2018). The Impella Device: Historical Background, Clinical Applications and Future Directions.International Journal of Angiology, 28 (02), 118-123.

2. Abiomed, Inc., (2020, June). Impella 5.5 With SmartAssist For Use During Cardiogenic Shock Instructions for Use and Clinical Reference Manual (Tech.). Retrieved September, 2020, from https://www.fda.gov/media/140766/download

3. Hong, E., \& Naseem, T. (2019). Color Doppler Artifact Masking Iatrogenic Aortic Valve Injury Related to an Impella Device. Journal of Cardiothoracic and Vascular Anesthesia,33 (6), 1584-1587. 
4. Nishimura, K., Iida, M., \& Shimokawa, T. (2020). Prosthetic valve replacement for aortic leaflet tear secondary to Impella device placement. Journal of Artificial Organs .

5. Oishi, H., Kondo, T., Fujimoto, K., et al, Murohara, T. (2020). Aortic insufficiency associated with Impella that required surgical intervention upon implantation of the durable left ventricular assist device. Journal of Artificial Organs .

6. Arias, E. A., Bhan, A., Lim, Z. Y., \& Mullen, M. (2019). TAVI for Pure Native Aortic Regurgitation: Are We There Yet? Interventional Cardiology Review, 14 (1), 26. 